\title{
Normal Values of High-resolution Manometry Parameters With Provocative Maneuvers
}

\author{
Hui Su, ${ }^{1}$ Amanda J Krause, ${ }^{2}$ Melina Masihi, ${ }^{2}$ Jacqueline Prescott, ${ }^{2}$ Alex Decorrevont, ${ }^{2}$ Emma Germond, ${ }^{2}$ Dave Karasik, ${ }^{2}$ \\ Wenjun Kou, ${ }^{2}$ John E Pandolfino, ${ }^{2}$ and Dustin A Carlson ${ }^{2 *}$ \\ ${ }^{\prime}$ Department of Gastroenterology, Capital Medical University Affiliated Beijing Shijitan Hospital, Beijing, China; and ${ }^{2}$ Division of \\ Gastroenterology and Hepatology, Department of Medicine, Feinberg School of Medicine, Northwestern University, Chicago, IL, USA
}

\section{Background/Aims}

Incorporation of complementary and provocative test swallows to the high-resolution manometry (HRM) protocol offers potential to address limitations posed by HRM protocols that involve only a single swallow type. The aim of this study is to describe normal findings of a comprehensive HRM testing protocol performed on healthy asymptomatic volunteers.

\section{Methods}

Thirty healthy asymptomatic volunteers completed HRM with 5-mL liquid swallows in the supine position. They also completed 5-mL liquid swallows in the upright position, viscous swallows, solid test swallows, multiple rapid swallows, and a rapid drink challenge. HRM studies were analyzed via Chicago classification version 3.0.

\section{Results}

The median (5th-95th percentiles) for integrated relaxation pressure (IRP) on supine swallows was 11 (4-16) $\mathrm{mmHg}$; IRP was lower than supine on upright liquid 9 (0-17) $\mathrm{mmHg}$, viscous $6(0-15) \mathrm{mmHg}$, solid 9 (1-19) $\mathrm{mmHg}$, multiple rapid swallows $3(0-12) \mathrm{mmHg}$, and rapid drink challenge $5(-3-12) \mathrm{mmHg} ; P<0.005$. While an "elevated" IRP value was observed on 1 to 2 test maneuvers in $8 / 30$ (27\%) subjects, all 30 subjects had an IRP value $<12 \mathrm{mmHg}$ on at least one of the test maneuvers.

\section{Conclusions}

Normal values and findings from a comprehensive HRM testing protocol are reported based on evaluation of 30 healthy asymptomatic volunteers. Isolated "abnormalities" of IRP and contractile parameters were observed in the majority (80\%) of these asymptomatic subjects, while all subjects also had normal features observed. Thus, the definition of "normal" should be recalibrated to focus on the entirety of the study and not individual metrics.

(J Neurogastroenterol Motil 2021;27:354-362)

\section{Key Words}

Manometry; Peristalsis; Reference values; Volunteers

Received: May 25, 2020 Revised: None Accepted: December 19, 2020

(a) This is an Open Access article distributed under the terms of the Creative Commons Attribution Non-Commercial License (http://creativecommons. org/licenses/by-nc/4.0) which permits unrestricted non-commercial use, distribution, and reproduction in any medium, provided the original work is properly cited.

*Correspondence: Dustin A Carlson, MD, MS

Division of Gastroenterology and Hepatology, Department of Medicine, Feinberg School of Medicine, Northwestern University, 676 St Clair St, Suite 1400, Chicago, IL 60611-2951, USA

Tel: +1-312-926-4939, Fax: +1-312-695-3999, E-mail: dustin-carlson@northwestern.edu 


\section{Introduction}

High-resolution manometry (HRM) is the primary method utilized in clinical practice to assess esophageal motility during the clinical evaluation of patients presenting with dysphagia, noncardiac chest pain, and other esophageal symptoms when upper gastrointestinal endoscopy does not reveal an objective diagnosis. ${ }^{1}$ The Chicago classification of esophageal motility disorders utilizes parameters generated from ten $5-\mathrm{mL}$ liquid test swallows performed in the supine position to categorize HRM findings into motility diagnoses. ${ }^{2,3}$ While the Chicago classification and its objective parameters of esophageal function reflects the common method to describe HRM studies and classify primary esophageal motility disorders using a standardized terminology, it is not without limitations. The Chicago classification may not adequately identify all abnormalities and also can sometimes yield false positive diagnoses. The classification of esophagogastric junction (EGJ) outflow obstruction is the primary example of the latter scenario in which the classification based on an integrated relaxation pressure (IRP) elevation among supine liquid test swallows may not consistently be clinically relevant. ${ }^{4-6}$

The Chicago classification presently is based upon a protocol of small-volume liquid swallows performed single position. This testing protocol may not be sufficient to detect all clinically relevant abnormalities, nor clarify when potential abnormal findings are spurious. Thus, incorporation of additional maneuvers into the manometric protocol, such as upright swallows, multiple rapid swallows (MRS), and a rapid drink challenge (RDC), offers the potential to improve the diagnostic yield of esophageal manometry. ${ }^{6-12}$ Establishing normal values related to these maneuvers is a necessary component to provide a comparative basis with patients undergoing clinical or research evaluation with HRM. Thus, the aim of this study is to report on parameters from adjunctive and provocative HRM maneuvers in asymptomatic volunteers.

\section{Materials and Methods}

\section{Subjects}

Healthy, asymptomatic (ie, free of esophageal symptoms including dysphagia, heartburn, and chest pain), adult volunteers were enrolled and completed between January 2017 and October 2019. Potential subjects were excluded for any previous diagnosis of esophageal, autoimmune, or eating disorders. Subjects were also excluded for use of antacids or proton pump inhibitors, body mass index $>30 \mathrm{~kg} / \mathrm{m}^{2}$, or a history of tobacco use or alcohol abuse. All subjects completed validated patient-reported outcomes including the brief esophageal dysphagia questionnaire (BEDQ), gastroesophageal reflux disease questionnaire (GERDQ), and esophageal hypervigilance and anxiety scale (EHAS), ${ }^{13-15}$ sedated upper endoscopy with functional luminal imaging probe, high-resolution impedance manometry, and 24-hour $\mathrm{pH}$-impedance testing. The HRM parameters were the focus of this report.

The study protocol was approved by the Northwestern University Institutional Review Board (STU00096856). Informed consent was obtained from all controls, who were paid for their participation. A portion of this cohort (20/30 subjects) was previously described. $^{16}$

\section{High-resolution Manometry}

Patients fasted for a minimum of 6 hours prior to completing HRM. The HRM studies used a 4.2-mm outer diameter solidstate assembly with 36 circumferential pressure sensors at $1-\mathrm{cm}$ intervals and 18 impedance segments at 2-cm intervals (Medtronic, Shoreview, MN, USA). The HRM device was placed transnasally to record from the hypopharynx to the stomach with approximately 3 intragastric pressure sensors.

The HRM protocol included 2-5 minutes of baseline recording. Liquid test swallows were performed using $50 \%$ saline for test swallows at 20-30 second intervals. The HRM protocol included ten 5 -mL liquid swallows in the supine position (Fig. 1). In the upright, seated position, subjects completed five 5 -mL liquid swallows, 5 viscous swallows (applesauce; completed in 24/30 [80\%] subjects), 2 solid swallows (chewed $3 \times 3 \mathrm{~cm}$ graham cracker), a MRS sequence, and a RDC. MRS involved drinking $2 \mathrm{~mL}$ of water for 5 successive swallows separated by 2-3 second intervals. ${ }^{9,17}$ The RDC involved drinking $200 \mathrm{~mL}$ of water with subjects instructed to drink as fast as possible. ${ }^{8,18-20}$

\section{Data Analysis}

ManoView version 3.0 (v3.0) analysis software (Medtronic, Shoreview, MN, USA) was used for analysis. The IRP, distal latency (DL), and distal contractile integral (DCI) were applied to test swallows per the Chicago classification. Individual swallow types were classified according to the Chicago classification v3.0 for all bolus types if DL was $>4.5$ seconds as effective (DCI 450$8000 \mathrm{mmHg} \cdot \mathrm{cm} \cdot \mathrm{sec}$ with no peristaltic break $>5 \mathrm{~cm}$ ); weak (DCI $100-450 \mathrm{mmHg} \cdot \mathrm{cm} \cdot \mathrm{sec}$ ), fragmented (DCI $>450 \mathrm{mmHg} \cdot \mathrm{cm} \cdot \mathrm{sec}$ and peristaltic break $>5 \mathrm{~cm}$ ), failed (DCI $<100 \mathrm{mmHg} \cdot \mathrm{cm} \cdot \mathrm{sec}$ ); 


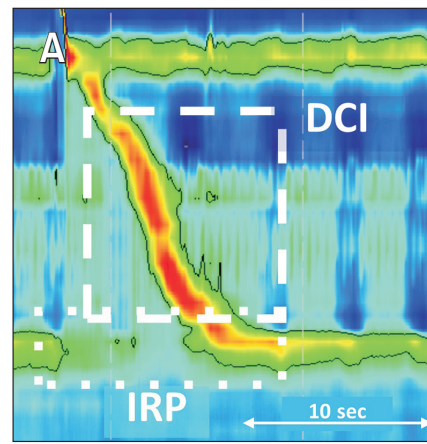

$22222 \mathrm{~mL}$

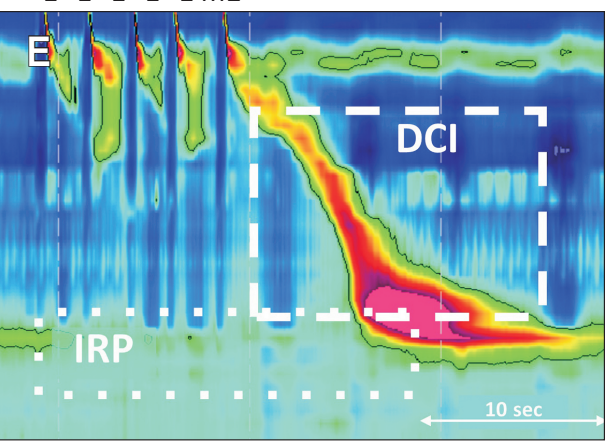

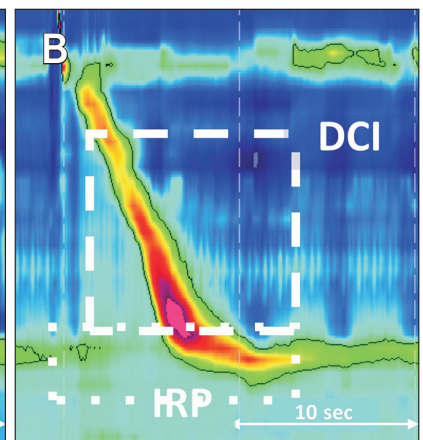
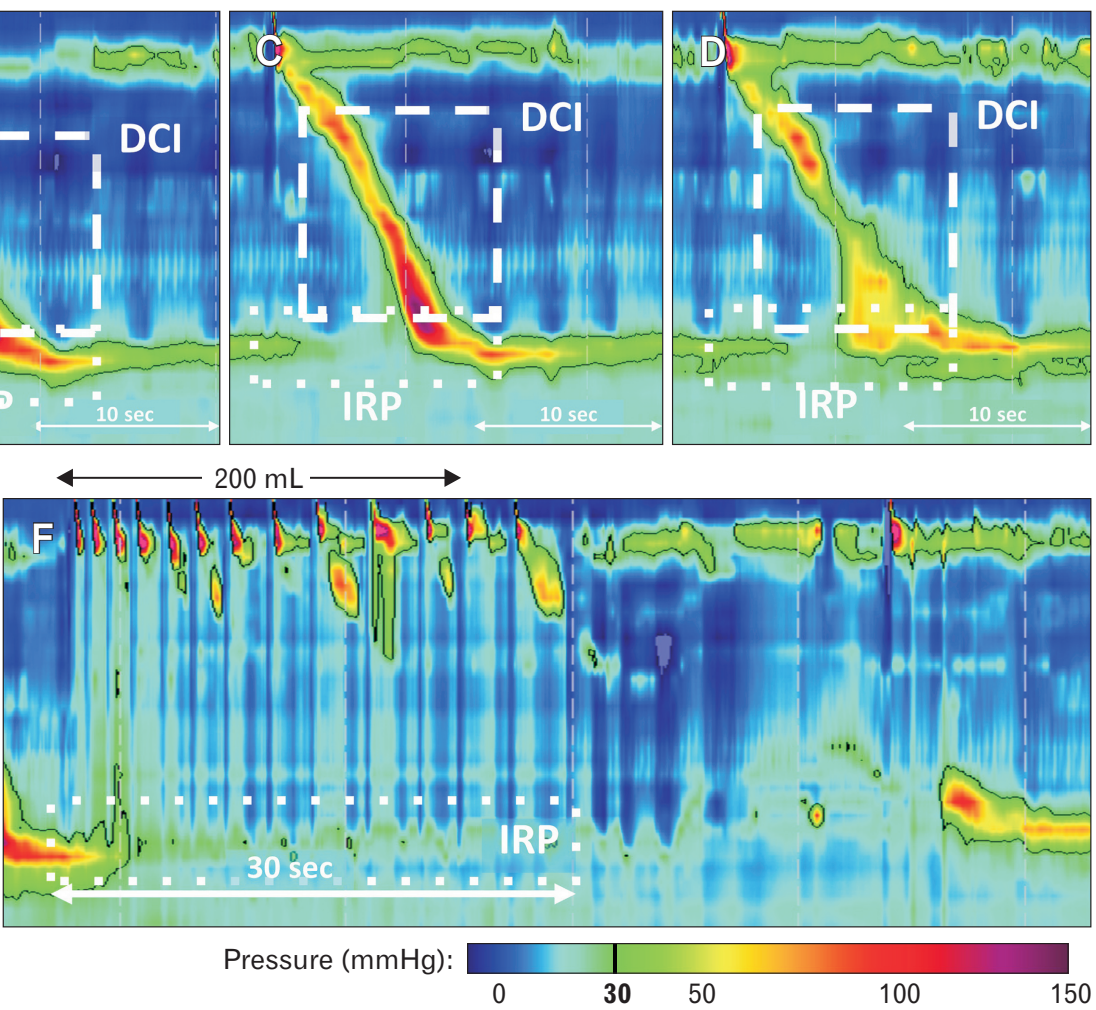

Figure 1. High-resolution manometry test swallows and maneuvers. (A) Supine $5 \mathrm{~mL}$ liquid; (B) upright $5 \mathrm{~mL}$ liquid; (C) viscous (apple sauce); (D) solid (graham cracker); (E) multiple rapid swallow; and (F) rapid drink challenge. Integrated relaxation pressure (IRP) and distal contractile integral (DCI) were $11 \mathrm{mmHg}$ and $1760 \mathrm{mmHg} \cdot \mathrm{cm} \cdot \mathrm{sec}(\mathrm{A}), 8 \mathrm{mmHg}$ and $1950 \mathrm{mmHg} \cdot \mathrm{cm} \cdot \mathrm{sec}(\mathrm{B}), 7 \mathrm{mmHg}$ and $1600 \mathrm{mmHg} \cdot \mathrm{cm} \cdot \mathrm{sec}(\mathrm{C}), 10$ $\mathrm{mmHg}$ and $1400 \mathrm{mmHg} \cdot \mathrm{cm} \cdot \mathrm{sec}(\mathrm{D}), 4 \mathrm{mmHg}$ and $4600 \mathrm{mmHg} \cdot \mathrm{cm} \cdot \mathrm{sec}(\mathrm{E})$, and $3 \mathrm{mmHg}$ and $0 \mathrm{mmHg} \cdot \mathrm{cm} \cdot \mathrm{sec}$ (F), respectively. Figure used with permission from the Esophageal Center at Northwestern.

and hypercontractile (> $8000 \mathrm{mmHg} \cdot \mathrm{cm} \cdot \mathrm{sec}){ }^{3}$ Weak, fragmented, and failed swallows were considered "ineffective." Premature swallows were classified if DL was $<4.5$ seconds and DCI > $450 \mathrm{mmHg} \cdot \mathrm{cm} \cdot \mathrm{sec}$. Failed swallows were analyzed with a DCI value of $0.0 \mathrm{mmHg} \cdot \mathrm{cm} \cdot \mathrm{sec}$ when applied to median DCI values. Esophageal motility classifications were derived in accord with the Chicago classification v3.0. ${ }^{3}$

During the baseline (resting) recording in the supine position, the intragastric pressure, EGJ morphology, basal EGJ pressure at end-expiration and end-inspiration, and the EGJ-contractile integral (EGJ-CI) were assessed. ${ }^{3,21}$ The EGJ-CI was measured by setting the isobaric contour to $2 \mathrm{mmHg}$ greater than the intragastric pressure then using the smart-mouse tool to measure the contractile integral over 3 respiratory cycles (then divided by the time of the measurement: thus into units of $\mathrm{mmHg} \cdot \mathrm{cm}){ }^{22}$

During MRS, the IRP was calculated over the entire duration of the sequence and MRS-DCI and MRS-swallow type were classified based on the peristaltic sequence following the final swal- low (Fig. 1). ${ }^{8,10}$ The MRS contractile augmentation was assessed by taking the MRS-DCI divided by the median-upright DCI. Contractile inhibition was also assessed during MRS and classified as "intact" when no contraction during the MRS sequence, or impaired when a contraction (DCI $>100 \mathrm{mmHg}$ and $>2 \mathrm{~cm}$ in axial length) occurred during the MRS sequence.

For RDC, several iterations of IRP measurement were assessed. The RDC-IRP was measured during the first 30 seconds of the RDC (reported as "RDC-IRP-30s"), over the entire RDCduration (reported as "RDC-IRP-total"; which equaled RDCIRP-30s if the RDC was completed in $<30$ seconds), and also as the averaged value of 10 -second increments over the entire duration of the RDC (reported as "RDC-IRP-AVG"). ${ }^{10}$ The duration of time to complete the RDC was also quantified and reported as "RDC-time." Esophageal-gastric (EG) gradient during RDC was measured as the difference between the mean esophageal pressure and mean gastric pressure $2 \mathrm{~cm}$ above and below the lower esophageal sphincter (LES), respectively. ${ }^{10}$ 
Table 1. Normal Values for Single Test Swallows

\begin{tabular}{|c|c|c|c|c|c|c|}
\hline Metric & $\begin{array}{c}\text { Supine } \\
5 \mathrm{~mL} \text { liquid }\end{array}$ & $\begin{array}{c}\text { Upright } \\
5 \mathrm{~mL} \text { liquid }\end{array}$ & $\begin{array}{l}\text { Viscous } \\
\text { swallows }\end{array}$ & $\begin{array}{c}\text { Solid } \\
\text { swallows }\end{array}$ & MRS & $\mathrm{RDC}^{\mathrm{a}}$ \\
\hline $\mathrm{n}$ & 30 & 30 & 26 & 29 & 30 & 30 \\
\hline $\operatorname{IRP}(\mathrm{mmHg})$ & $11(4-16)$ & $9(0-17)^{\mathrm{b}}$ & $6(0-15)^{\mathrm{b}, \mathrm{c}}$ & $9(1-19)^{b}$ & $3(0-12)^{b, c}$ & $5(-3-12)^{b, c}$ \\
\hline Median DCI $(\mathrm{mmHg} \cdot \mathrm{cm} \cdot \mathrm{sec})$ & $1747(366-6335)$ & $1578(94-5110)^{b}$ & $1689(331-5125)^{\mathrm{b}}$ & $2848(0-4428)$ & - & - \\
\hline Greatest DCI $(\mathrm{mmHg} \cdot \mathrm{cm} \cdot \mathrm{sec})$ & $2295(566-10980)$ & $2257(350-6896)^{\mathrm{b}}$ & $2276(746-7200)^{\mathrm{b}}$ & $2231(0-5153)$ & $2645(0-10362)$ & - \\
\hline Shortest DL (sec) & $6.0(4.9-8.0)$ & $6.2(4.6-8.2)$ & $7.0(5.2-9.1)^{\mathrm{b}, \mathrm{c}}$ & $6.5(4.8-8.7)^{\mathrm{b}, \mathrm{c}}$ & - & - \\
\hline
\end{tabular}

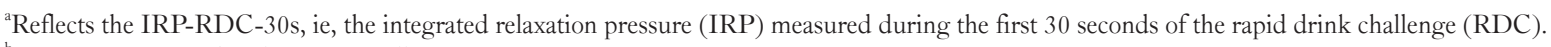

${ }^{\mathrm{b}} P<0.05$ compared with supine swallows.

${ }^{\mathrm{c}} \mathrm{P}<0.05$ compared with upright, $5 \mathrm{~mL}$ liquid swallows.

MRS, multiple rapid swallows; DCI, distal contractile integral; DL, distal latency.

Viscous and solid swallows, as well as MRS and RDC were performed in the upright, seated position.

Values reflect median (5th-95th percentiles).

Presence of panesophageal pressurization, defined as continuous pressurization from the upper esophageal sphincter to the EGJ at an isobaric contour of $30 \mathrm{mmHg}$ was also assessed during MRS and RDC.

\section{Statistical Methods}

Data were analyzed using SPSS version 26 (IBM Corporation, Armonk, NY, USA). To maintain data independence, data from each individual subject were analyzed by taking the median, maximum (greatest), of minimum (smallest) value across swallows of similar bolus and position. Data are expressed as median (5th95th percentiles) or interquartile range (IQR) or mean (SD) based on data distribution. Comparisons were made using Wilcoxon rank sum or paired $t$ tests, based on data distribution. Correlation was assessed using Spearman's rho. Analyses assumed a 5\% level of statistical significance.

\section{Results}

\section{Study Subjects}

The 30 asymptomatic controls had a mean (SD) age of 30 (6) years and $76 \%$ were female. The mean (SD) body mass index was 23 (3) $\mathrm{kg} / \mathrm{m}^{2}$. The controls all had a normal upper endoscopy. The BEDQ score was 0 in 29/30 controls (and 1 in the other); the GERDQ score was normal $(<9)$ in all 30 subjects; the EHAS score was 0 in 28/30 ( 8 and 24 in the other 2 , respectively). Twentyfour hour $\mathrm{pH}$-impedance was completed in $27 / 30$ and total acid exposure time for $\mathrm{pH}<4$ was median (5th-95th) $0.7 \%$ (0.0-6.0); one subject had an acid exposure time $>6.0 \%(6.3 \%)$.

\section{Resting Esophagogastric Junction Evaluation}

The EGJ morphology was type I in 28/30 (94\%) subjects and type II in 2 (with $1 \mathrm{~cm}$ of LES-crural separation). The intragastric pressure was mean (5th-95th) 8 (1-16) mmHg. The basal EGJ pressure at end-expiration was median (5th-95th) 15 (4-32) $\mathrm{mmHg}$ and at end-inspiration was median (5th-95th) 23 (10-55) $\mathrm{mmHg}$. The EGJ-CI was median (5th-95th) $44(9-141) \mathrm{mmHg} \cdot \mathrm{cm}$. The end-expiratory basal EGJ pressure and EGJ-CI were strongly correlated: $r h o=0.910(P<0.001)$ (Supplementary Figure).

\section{Liquid Test Swallows}

On supine $5 \mathrm{~mL}$ liquid swallows, the median (5th-95th) supine IRP was 11 (4-16) mmHg (Table 1). Esophageal motility classification was normal esophageal motility in 26 (87\%), ineffective esophageal motility (IEM) in 2 (7\%), and EGJ outflow obstruction in $2(7 \%)$. EGJ outflow obstruction subjects had median supine IRP values of $16 \mathrm{mmHg}$ and $17 \mathrm{mmHg}$ and both had normal peristalsis. The 2 subjects with IEM had 8/10 and 10/10 ineffective swallows and median DCI values of 760 and $194 \mathrm{mmHg} \cdot \mathrm{cm} \cdot \mathrm{sec}$, respectively. There were 2 subjects that each had 1 hypercontractile swallow among the 10 supine swallows; there were no premature swallows observed.

On upright $5 \mathrm{~mL}$ liquid swallows, the median (5th-95th) IRP was 9 (0-17) $\mathrm{mmHg}$, which was lower than on supine swallows $(P<0.001)$ (Fig. 2). The 2 controls with supine IRP $>15 \mathrm{mmHg}$ had upright IRPs of $10 \mathrm{mmHg}$ and $14 \mathrm{mmHg}$, respectively. Both subjects with IEM on supine swallows also had IEM on upright swallows ( $5 / 5$ ineffective upright swallows in both); there was 5 additional subjects with $>50 \%$ ineffective upright swallows. Application of Chicago classification concepts to upright swallows with 


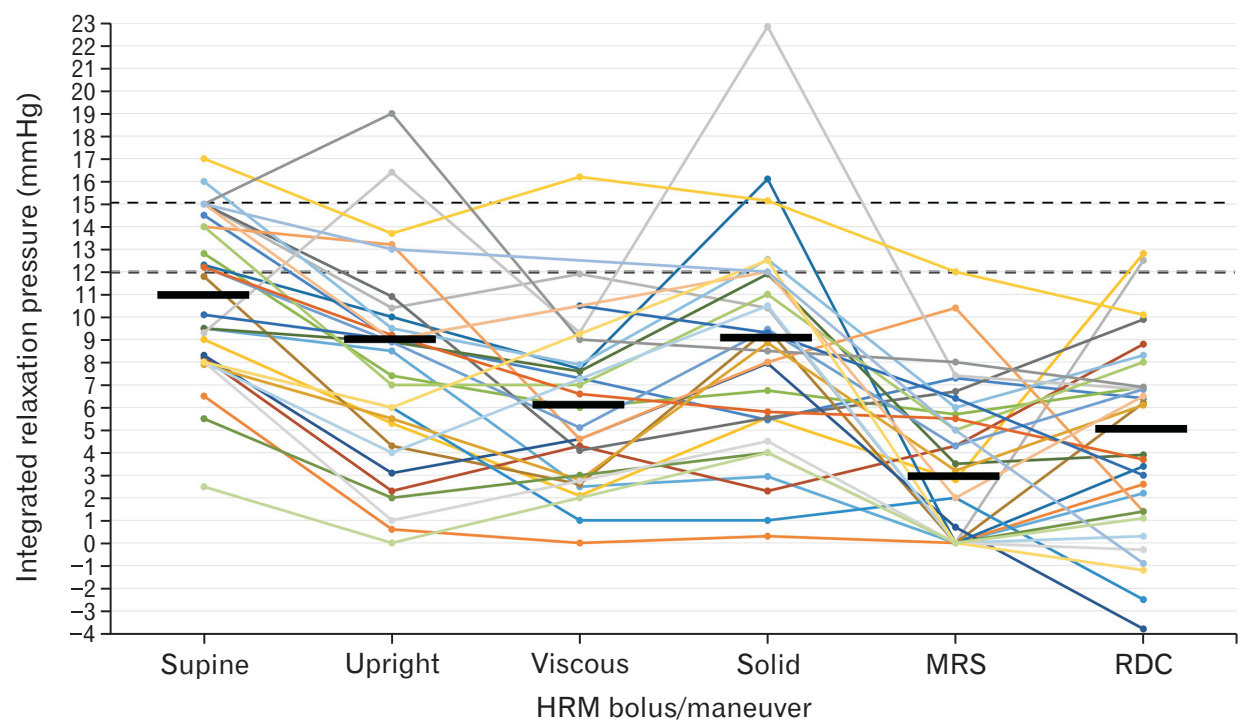

Figure 2. Integrated relaxation pressure (IRP) values by manometric test swallow/maneuver. Each line represents one asymptomatic volunteer. Common thresholds of $12 \mathrm{mmHg}$ and $15 \mathrm{mmHg}$ are reflected by the dashed horizontal lines. Median values for each swallow type or maneuver are represented by a solid horizontal line. The rapid drink challenge (RDC)-IRP value reflects the IRP measured during the first 30 seconds of the RDC ("RDC-IRP-30s"). HRM, high-resolution manometry; MRS, multiple rapid swallows. Figure used with permission from the Esophageal Center at Northwestern.

an upper limit of normal IRP threshold of $12 \mathrm{mmHg}$ to this cohort yielded 5/30 (17\%) subjects with EGJ outflow obstruction, (one of whom had ineffective peristalsis), 7/30 (23\%) with IEM, and $18 / 30(60 \%)$ with normal motility. There were no hypercontractile nor premature swallows observed among the upright $5 \mathrm{~mL}$ liquid swallows.

\section{Viscous and Solid Swallows}

Twenty-six subjects completed 5 viscous swallows in the upright position, and 29 completed 2 solid swallows (1 had gluten sensitivity and declined). Median IRP values were lower than the median IRP from supine liquid swallows on paired comparisons $(P<0.001$ and $P=0.005$, respectively) (Table 1 and Fig. 2). Compared with supine liquid swallows, median and greatest DCI values were lower with viscous swallows, but similar with the solid swallows. There were 2 subjects with $>50 \%$ ineffective swallows among the viscous swallows; neither of which had "IEM" on supine or upright $5 \mathrm{~mL}$ liquid swallows. All but 1 subject (28/29) had 1 or 2 effective solid swallows; this 1 subject had IEM on both supine and upright swallows, a failed swallow after MRS, and did not complete viscous swallows. One subject had a single hypercontractile swallow on viscous swallows; no hypercontractile swallows were observed with solid swallows. No premature swallows were observed on viscous or solid swallows.

\section{Multiple Rapid Swallows and Rapid Drink Challenge}

On MRS, the median (5th-95th) IRP was 3 (0-12) $\mathrm{mmHg}$, which was lower than the median supine IRP $(P<0.001)$. The peristaltic wave after MRS was classified as normal in $20(67 \%)$, hypercontractile in $3(10 \%)$, weak in $3(10 \%)$, and failed in 4 (13\%). The MRS-DCI had a median (5th-95th) of 2287 (0-9459) $\mathrm{mmHg} \cdot \mathrm{cm} \cdot \mathrm{sec}$. The augmentation ratio (MRS-DCI/median upright DCI) was a median (5th-95th) 1.9 (0-13.6); 21/30 (70\%) had an MRS-augmentation ratio of $\geq 1.0$, ie, contractile reserve. Among the 9 patients with $>50 \%$ ineffective swallows ("IEM") on either supine $(\mathrm{n}=2)$, upright $(\mathrm{n}=7 ; 2$ also with supine IEM), or viscous swallows $(\mathrm{n}=2), 6(67 \%)$ had contractile reserve observed after MRS. Contractile inhibition on MRS was impaired in $6 / 30(20 \%)$.

The RDC was completed in a median (5th-95th) 39 (11-159) seconds. The median (5th-95th) RDC-IRP-30s was 5 (-3-12) mmHg. The median (5th-95th) RDC-IRP-total was 3 (-3-12) $\mathrm{mmHg}$, while the median (5th-95th) RDC-IRP-AVG (ie, averaged 10-second intervals) was $6(-2-16) \mathrm{mmHg}$. All RDC-IRP measures were lower than the median supine $\operatorname{IRP}(P<0.001)$. The median (5th-95th) RDC-EG-gradient was 2.3 (-6-10) $\mathrm{mmHg}$. Pan-esophageal pressurization was observed during the RDC in 1 subject that had an RDC-IRP-30s of $3 \mathrm{mmHg}$. 
Table 2. Abnormal Findings Among Asymptomatic Volunteers

\begin{tabular}{|c|c|c|c|c|c|c|c|c|c|}
\hline \multicolumn{6}{|c|}{ IRP (mmHg) } & \multicolumn{4}{|c|}{ Contractility (pattern) } \\
\hline Supine & Upright & Viscous & Solid & MRS & RDC & Supine & Upright & Viscous & MRS \\
\hline 17 & 14 & 16 & 15 & 12 & 10 & Normal & Normal & Normal & Normal \\
\hline 16 & 10 & 8 & 13 & 6 & 8 & Normal & Normal & Normal & Normal \\
\hline 15 & 19 & 9 & - & 8 & 7 & Normal & Normal & Normal & Normal \\
\hline 15 & 13 & - & 12 & 5 & -1 & Normal & Normal & - & Normal \\
\hline 14 & 13 & 5 & 8 & 10 & 1 & Normal & Normal & $\mathrm{HC}$ & Im-CR \\
\hline 9 & 16 & 9 & 23 & 7 & 7 & $\mathrm{HC}$ & Normal & Normal & Normal \\
\hline 15 & 11 & 4 & 6 & 7 & 10 & Normal & IEM & Normal & Normal \\
\hline 15 & 10 & 12 & 10 & 0 & 13 & Normal & Normal & Normal & Im-INH \\
\hline 15 & 9 & 7 & 5 & 7 & 6 & IEM & IEM & Normal & Normal \\
\hline 14 & 7 & 7 & 11 & 5 & 8 & Normal & Normal & Normal & Im-CR \\
\hline 12 & 10 & 8 & 16 & 0 & 3 & $\mathrm{HC}$ & Normal & Normal & Im-INH \\
\hline 12 & 9 & 7 & 6 & 6 & 4 & Normal & Normal & IEM & $\begin{array}{l}\text { Im-CR } \\
\text { Im-INH }\end{array}$ \\
\hline 12 & 9 & 5 & 9 & 4 & 7 & Normal & Normal & Normal & $\mathrm{Im}-\mathrm{CR}$ \\
\hline 12 & 4 & 3 & 9 & 0 & 6 & Normal & Normal & Normal & Im-INH \\
\hline 10 & 9 & 11 & 9 & 6 & 3 & Normal & Normal & Normal & $\begin{array}{l}\mathrm{Im}-\mathrm{CR} \\
\mathrm{Im}-\mathrm{INH}\end{array}$ \\
\hline 10 & 9 & 3 & 3 & 0 & 2 & Normal & Normal & Normal & $\begin{array}{l}\mathrm{Im}-\mathrm{CR} \\
\mathrm{Im}-\mathrm{INH}\end{array}$ \\
\hline 9 & 5 & 2 & 6 & 3 & 13 & Normal & Normal & Normal & Normal \\
\hline 8 & 6 & 1 & 1 & 2 & -3 & Normal & Normal & Normal & Im-CR \\
\hline 8 & 6 & - & 13 & 0 & -1 & Normal & IEM & - & Normal \\
\hline 8 & 4 & - & 11 & 0 & 0 & IEM & IEM & - & Im-CR \\
\hline 8 & 1 & - & 5 & 0 & 0 & Normal & IEM & & Normal \\
\hline 8 & 6 & 3 & 9 & 3 & 6 & Normal & Normal & IEM & Normal \\
\hline 6 & 2 & 3 & 4 & 0 & 1 & Normal & IEM & Normal & Im-CR \\
\hline 3 & 0 & - & 4 & 0 & 1 & Normal & IEM & - & Normal \\
\hline 15 & 9 & - & 12 & 2 & 7 & Normal & Normal & - & Normal \\
\hline 10 & 9 & 8 & 12 & 4 & 4 & Normal & Normal & Normal & Normal \\
\hline 8 & 3 & 5 & 8 & 1 & -4 & Normal & Normal & Normal & Normal \\
\hline 8 & 2 & 4 & 2 & 4 & 9 & Normal & Normal & Normal & Normal \\
\hline 7 & 1 & 0 & 0 & 0 & 3 & Normal & Normal & Normal & Normal \\
\hline 13 & 7 & 6 & 7 & 6 & 7 & Normal & Normal & Normal & Normal \\
\hline
\end{tabular}

MRS, multiple rapid swallows; RDC, rapid drink challenge; HC, hypercontractile swallow; Im-CR, impaired contractile reserve; IEM, ineffective esophageal motility; Im-INH, impaired inhibition during MRS.

Each row represents one asymptomatic volunteer. Findings considered "abnormal" are highlighted in gray: "Elevated" integrated relaxation pressure (IRP) was considered if the median value among test swallows was $>15 \mathrm{mmHg}$ for supine $5 \mathrm{~mL}$ liquid swallows, viscous, or solid test swallows or $>12 \mathrm{mmHg}$ for upright $5 \mathrm{~mL}$ liquid swallows, MRS, or RDC. IEM was considered if $>50 \%$ of test swallows were ineffective.

The 2 controls with supine IRP $>15 \mathrm{mmHg}$ had MRS-IRPs of $6 \mathrm{mmHg}$ and $12 \mathrm{mmHg}$, RDC-IRP-30s of $8 \mathrm{mmHg}$ and 10 $\mathrm{mmHg}$, and RDC-EG-gradients of $2 \mathrm{mmHg}$ and $5 \mathrm{mmHg}$. None of the controls had an MRS-IRP $>12 \mathrm{mmHg}$. Two of 30 (7\%) had RDC-IRP > $12 \mathrm{mmHg}$ by RDC-IRP-30s, RDCIRP-total, and RDC-IRP-AVG; a third subject had only RDCIRP-AVG $>12 \mathrm{mmHg}$ (but $<12$ for both RDC-IRP-30s and RDC-IRP-total).
Findings potentially considered as "abnormal" were observed during the various test swallows and maneuvers among 24 of these 30 (80\%) asymptomatic volunteers (Table 2). Conversely, all 30 subjects had an IRP $<12 \mathrm{mmHg}$ on at least 1 of the test maneuvers (Fig. 2) and 29/30 (97\%) subjects had normal motility (> 50\% effective swallows) on either supine, upright, or viscous swallows. 


\section{Discussion}

We evaluated 30 healthy asymptomatic volunteers with HRM and a testing protocol that included supine liquid swallows, upright liquid swallows, viscous swallows, single solid test swallows, MRS, and RDC. The normal values derived from this cohort provide comparison for clinical application to patients tested with comprehensive HRM protocols. Additionally, we observed findings that could be considered "abnormal" in $70 \%$ of these healthy asymptomatic volunteers; however these were generally isolated findings and otherwise normal findings were observed on other portions of the testing protocols.

Various factors related to the manometric test protocol contribute to the quantifiable objective parameters that are associated with esophageal motility evaluation with HRM: these include bolus consistency, bolus volume, subject position, and manufacturer of HRM assembly. ${ }^{23}$ Thus, consistency in HRM study protocol and appropriate application of normal values derived from similar testing situations are important for clinical utilization. The present study evaluated multiple components that could be incorporated into a comprehensive HRM testing protocol and observed fairly consistent parameters as those reported with previous studies of asymptomatic volunteers. ${ }^{8,1,11,21,23-28}$

Incorporation of the provocative maneuvers the MRS and $\mathrm{RDC}$ have recently gained additional interest for clinical manometric evaluation of both gastroesophageal reflux disease and obstructive esophageal disorders. ${ }^{8,9,17,18,20,27-29}$ The present study supports the IRP threshold of $12 \mathrm{mmHg}$ reported in several studies assessing the RDC. ${ }^{10,11}$ The present study also observed that similar methods to measure IRP during RDC resulted in similar output; thus application of the IRP-window over the initial 30 seconds of the RDC may provide an acceptable balance of accuracy with ease of application. The MRS has generally been utilized to assess for contractile reserve and was observed with a similar prevalence of contractile reserve in the present study compared with previous studies (70-80\% of healthy controls). ${ }^{9,30}$ Assessment of LES relaxation during MRS using the IRP may also be of clinical utility and the upper limit of normal of $12 \mathrm{mmHg}$ reported in this study is the same as reported in another study. ${ }^{30}$ The MRS has also been applied to assess contractile inhibition during MRS, observed, however in $20 \%$ of this asymptomatic cohort and 2/18 healthy controls (11\%) in a previous study. ${ }^{31}$

The IRP garners particular attention given the importance placed on it as the initial decision point within the hierarchical
Chicago classification scheme, primarily the $15 \mathrm{mmHg}$ threshold when using supine $5 \mathrm{~mL}$ swallows with a Sierra-vintage HRM assembly. ${ }^{3}$ The $12 \mathrm{mmHg}$ threshold has been previous applied with upright liquid swallows with clinical relevance. ${ }^{5,6}$ While the upright IRP was lower than supine swallows for the majority of subjects in this study as expected similar to previous studies, ${ }^{23,24}$ the upper limit of the upright IRP observed here was driven primarily by 2 outlying values (Fig. 2): one was likely related to a small reducible hiatal hernia that was not appreciated endoscopically (type II EGJ morphology); an explanation for the other can be speculated as potential catheter impingement artifact generated by the position change. ${ }^{32}$ When incorporating all of the numerous test swallows/maneuvers included in this testing protocol (ie, supine, upright, viscous, solid, MRS, and RDC) there were 8/30 (27\%) asymptomatic healthy subjects with an IRP value that would be considered elevated based on previously applied thresholds of $15 \mathrm{mmHg}$ (supine swallows) or $12 \mathrm{mmHg}$ (upright liquid swallows or RDC) (Table 2). ${ }^{3,10,11,24}$ However, the intra-individual fluctuation of IRP values between these various maneuvers (Fig. 2) demonstrated that elevated IRP values were generally isolated to a single test scenario (only 2 subjects had IRP values greater than previously applied thresholds on more than 1 bolus type/maneuver). Similarly, while contractile abnormalities (particular $>50 \%$ ineffective swallows suggesting "IEM") were observed, all but 1 subject demonstrated effective peristalsis during at least 1 of the test maneuvers.

While this study describes 30 well-characterized asymptomatic volunteers with a comprehensive HRM protocol, it does carry some limitations. As is common among studies assessing healthy asymptomatic volunteers, the cohort evaluated was relatively young and thus changes in esophageal motility that are associated with the normal aging process may not be reflected by this cohort. Additionally, while a comprehensive HRM test protocol was assessed, there are other promising HRM maneuvers, such as the solid test meal, that were not incorporated into this study's protocol. ${ }^{11,33}$ Additionally, parameters that incorporate impedance (high-resolution impedance manometry) may also offer benefit to the HRM evaluation, however, assessing combined manometry-impedance measures was not the focus of this evaluation.

In summary, HRM parameters of healthy, asymptomatic volunteers associated with supine liquid swallows (the current standard Chicago classification protocol), as well as upright liquid swallows, viscous swallows, solid test swallows, MRS, and RDC were described. These values provide a reference for comparison for clinical application of symptomatic patients. Additionally, it should be noted that isolated "abnormalities" of both IRP and contractile param- 
eters were observed in the majority of these healthy asymptomatic controls. This demonstrates that as the HRM protocol expands to include numerous additional maneuvers, caution needs to be exercised when interpreting the clinical relevance of isolated "abnormalities." Instead, consistently abnormal findings across multiple test maneuvers should be present to support clinically-relevant esophageal motility abnormalities; future studies of patients to support this approach remain necessary.

\section{Supplementary Materials}

Note: To access the supplementary figure mentioned in this article, visit the online version of Journal of Neurogastroenterology and Motility at http://www.jnmjournal.org/, and at https://doi. org/10.5056/jnm20118.

Financial support: This work was supported by the Public Health service (P01 DK117824 (JEP) and American College of Gastroenterology Junior Faculty Development Award (D.A.C.).

Conflicts of interest: Wenjun Kou: Crospon, Inc (consulting); John E Pandolfino: Crospon, Inc (stock options), Given Imaging (consultant, grant, and speaking), Sandhill Scientific (consulting and speaking), Takeda (speaking), Astra Zeneca (speaking), Medtronic (speaking and consulting), Torax (speaking and consulting), Ironwood (consulting), and Impleo (grant); and Dustin A Carlson: Medtronic (speaking and consulting). Hui Su, Amanda J Krause, Melina Masihi Jacqueline Prescott, Alex Decorrevont, Emma Germond, and Dave Karasik have no conflicts of interest.

Author contributions: Hui Su contributed to data analysis, data interpretation, drafting of the manuscript, and approval of the final version; Amanda J Krause, Jacqueline Prescott, Alex Decorrevont, Emma Germond, Dave Karasik, and Wenjun Kou contributed to data analysis and approval of the final version; Melina Masihi contributed to recruitment of subjects, data analysis, and approval of the final version; John E Pandolfino contributed to study concept and design, obtaining funding, editing the manuscript critically, and approval of the final version; and Dustin A Carlson contributed to study concept and design, data analysis, data interpretation, drafting of the manuscript, and approval of the final version.

\section{References}

1. Pandolfino JE, Kahrilas PJ. American gastroenterological association medical position statement: clinical use of esophageal manometry. Gastro- enterology 2005;128:207-208

2. Pandolfino JE, Ghosh SK, Rice J, Clarke JO, Kwiatek MA, Kahrilas PJ. Classifying esophageal motility by pressure topography characteristics: a study of 400 patients and 75 controls. Am J Gastroenterol 2008;103:2737.

3. Kahrilas PJ, Bredenoord AJ, Fox M, et al. The Chicago classification of esophageal motility disorders, v3.0. Neurogastroenterol Motil 2015;27:160-174.

4. van Hoeij FB, Smout AJ, Bredenoord AJ. Characterization of idiopathic esophagogastric junction outflow obstruction. Neurogastroenterol Motil 2015;27:1310-1316.

5. Babaei A, Shad S, Szabo A, Massey BT. Pharmacologic interrogation of patients with esophagogastric junction outflow obstruction using amyl nitrite. Neurogastroenterol Motil 2019;31:e13668.

6. Triggs JR, Carlson DA, Beveridge C, et al. Upright integrated relaxation pressure facilitates characterization of esophagogastric junction outflow obstruction. Clin Gastroenterol Hepatol 2019;17:2218-2226, e2.

7. Carlson DA, Roman S. Esophageal provocation tests: are they useful to improve diagnostic yield of high resolution manometry? Neurogastroenterol Motil 2018;30:e13321.

8. Ang D, Hollenstein M, Misselwitz B, et al. Rapid drink challenge in high-resolution manometry: an adjunctive test for detection of esophageal motility disorders. Neurogastroenterol Motil 2017;29:e12902.

9. Shaker A, Stoikes N, Drapekin J, Kushnir V, Brunt LM, Gyawali CP. Multiple rapid swallow responses during esophageal high-resolution manometry reflect esophageal body peristaltic reserve. Am J Gastroenterol 2013;108:1706-1712.

10. Marin I, Cisternas D, Abrao L, et al. Normal values of esophageal pressure responses to a rapid drink challenge test in healthy subjects: results of a multicenter study. Neurogastroenterol Motil 2017;29:e13021.

11. Misselwitz B, Hollenstein M, Butikofer S, Ang D, Heinrich H, Fox M. Prospective serial diagnostic study: the effects of position and provocative tests on the diagnosis of oesophageal motility disorders by high-resolution manometry. Aliment Pharmacol Ther 2020;51:706-718.

12. Singendonk MJ, Lin Z, Scheerens C, et al. High-resolution impedance manometry parameters in the evaluation of esophageal function of non-obstructive dysphagia patients. Neurogastroenterol Motil 2019;31:e13505.

13. Taft TH, Riehl M, Sodikoff JB, et al. Development and validation of the brief esophageal dysphagia questionnaire. Neurogastroenterol Motil 2016;28:1854-1860.

14. Jonasson C, Wernersson B, Hoff DA, Hatlebakk JG. Validation of the GerdQ questionnaire for the diagnosis of gastro-oesophageal reflux disease. Aliment Pharmacol Ther 2013;37:564-572.

15. Taft TH, Triggs JR, Carlson DA, et al. Validation of the oesophageal hypervigilance and anxiety scale for chronic oesophageal disease. Aliment Pharmacol Ther 2018;47:1270-1277.

16. Carlson DA, Kou W, Lin Z, et al. Normal values of esophageal distensibility and distension-induced contractility measured by functional luminal imaging probe panometry. Clin Gastroenterol Hepatol 2019;17:674-681, e1.

17. Fornari F, Bravi I, Penagini R, Tack J, Sifrim D. Multiple rapid swal- 
lowing: a complementary test during standard oesophageal manometry. Neurogastroenterol Motil 2009;21:718-e41.

18. Daum C, Sweis R, Kaufman E, et al. Failure to respond to physiologic challenge characterizes esophageal motility in erosive gastro-esophageal reflux disease. Neurogastroenterol Motil 2011;23:517-e200.

19. Elvevi A, Mauro A, Pugliese D, et al. Usefulness of low-and highvolume multiple rapid swallowing during high-resolution manometry. Dig Liver Dis 2015;47:103-107.

20. Marin I, Serra J. Patterns of esophageal pressure responses to a rapid drink challenge test in patients with esophageal motility disorders. Neurogastroenterol Motil 2016;28:543-553.

21. Pandolfino JE, Ghosh SK, Zhang Q, Jarosz A, Shah N, Kahrilas PJ. Quantifying EGJ morphology and relaxation with high-resolution manometry: a study of 75 asymptomatic volunteers. Am J Physiol Gastrointest Liver Physiol 2006;290:G1033-G1040.

22. Nicodème F, Pipa-Muniz M, Khanna K, Kahrilas PJ, Pandolfino JE. Quantifying esophagogastric junction contractility with a novel HRM topographic metric, the EGJ-contractile integral: normative values and preliminary evaluation in PPI non-responders. Neurogastroenterol Motil 2014;26:353-360.

23. Herregods TV, Roman S, Kahrilas PJ, Smout AJ, Bredenoord AJ. Normative values in esophageal high-resolution manometry. Neurogastroenterol Motil 2015;27:175-187.

24. Xiao Y, Read A, Nicodème F, Roman S, Kahrilas PJ, Pandolfino JE. The effect of a sitting vs supine posture on normative esophageal pressure topography metrics and Chicago classification diagnosis of esophageal motility disorders. Neurogastroenterol Motil 2012;24:e509-e516.

25. Sweis R, Anggiansah A, Wong T, Kaufman E, Obrecht S, Fox M. Normative values and inter-observer agreement for liquid and solid bolus swallows in upright and supine positions as assessed by esophageal high- resolution manometry. Neurogastroenterol Motil 2011;23:509-e198.

26. Ghosh SK, Pandolfino JE, Zhang Q, Jarosz A, Shah N, Kahrilas PJ. Quantifying esophageal peristalsis with high-resolution manometry: a study of 75 asymptomatic volunteers. Am J Physiol Gastrointest Liver Physiol 2006;290:G988-G997.

27. Kushnir V, Sayuk GS, Gyawali CP. Multiple rapid swallow responses segregate achalasia subtypes on high-resolution manometry. Neurogastroenterol Motil 2012;24:1069-e561.

28. Omari TI, Wauters L, Rommel N, Kritas S, Myers JC. Oesophageal pressure-flow metrics in relation to bolus volume, bolus consistency, and bolus perception. United European Gastroenterol J 2013;1:249-258.

29. Biasutto D, Mion F, Garros A, Roman S. Rapid drink challenge test during esophageal high resolution manometry in patients with esophago-gastric junction outflow obstruction. Neurogastroenterol Motil 2018;30:e13293.

30. Leopold A, Yu D, Bhuta R, et al. Multiple rapid swallows (MRS) complements single-swallow (SS) analysis for high-resolution esophageal manometry (HREM). Dig Dis Sci 2019;64:2206-2213.

31. Quader F, Mauro A, Savarino E, et al. Jackhammer esophagus with and without esophagogastric junction outflow obstruction demonstrates altered neural control resembling type 3 achalasia. Neurogastroenterol Motil 2019;31:e13678.

32. Babaei A, Szabo A, Yorio SD, Massey BT. Pressure exposure and catheter impingement affect the recorded pressure in the Manoscan $360^{\mathrm{TM}}$ system. Neurogastroenterol Motil 2018;30:e13329.

33. Ang D, Misselwitz B, Hollenstein M, et al. Diagnostic yield of highresolution manometry with a solid test meal for clinically relevant, symptomatic oesophageal motility disorders: serial diagnostic study. Lancet Gastroenterol Hepatol 2017;2:654-661. 